\title{
Single colloidal quantum dots as sources of single photons for quantum cryptography
}

\author{
Ferruccio Pisanello*a, ${ }^{\text {a, }}$, Antonio Qualtieri ${ }^{\mathrm{a}, \mathrm{c}}$, Godefroy Leménager ${ }^{\mathrm{b}}$, Luigi Martiradonna ${ }^{\mathrm{a}, \mathrm{c}}$, Tiziana \\ Stomeo ${ }^{\mathrm{a}}$, Roberto Cingolani ${ }^{\mathrm{a}, \mathrm{c}}$, Alberto Bramati ${ }^{\mathrm{b}}$, Massimo De Vittorio* ${ }^{\mathrm{a}, \mathrm{c}}$ \\ ${ }^{a}$ National Nanotechnology Laboratory, CNR-Istituto di Nanoscienze, Scuola superiore ISUFI, \\ Università del Salento, 16 Via Arnesano, Lecce 73100, Italy; \\ ${ }^{\mathrm{b}}$ Université Pierre et Marie Curie, Laboratoire Kastler Brossel, CNRS UMR8552, Ecole Normale \\ Supérieure, 4 place Jussieu, 75252 Paris Cedex 05, France \\ ${ }^{\mathrm{c}}$ Center for Bio-Molecular Nanotechnology, Istituto Italiano di Tecnologia (IIT), Via Barsanti 1, \\ Arnesano, Lecce 73010, Italy
}

\begin{abstract}
Colloidal nanocrystals, i.e. quantum dots synthesized trough wet-chemistry approaches, are promising nanoparticles for photonic applications and, remarkably, their quantum nature makes them very promising for single photon emission at room temperature. In this work we describe two approaches to engineer the emission properties of these nanoemitters in terms of radiative lifetime and photon polarization, drawing a viable strategy for their exploitation as room-temperature single photon sources for quantum information and quantum telecommunications.
\end{abstract}

Keywords: quantum dots, colloidal nanocrystals, single photon source

\section{INTRODUCTION}

Quantum information and quantum telecommunications are strategic research fields in which the fundamental laws of the quantum physics are exploited to implement new protocols able to overtake the limitations of classical communications. One of the major drawback of classical systems is provided in the cryptographic key exchange procedures: commercial classical protocols for the key distribution are based on public-key encryption, in which the security is based on the difficulty in inverting the mathematical functions used to encrypt the message. These schemes can, in principle, be violated with some innovative algorithm or by the development of powerful quantum computers. Even if Quantum Keys Distribution (QKD) can be realized with attenuated coherent beams, the use of single photons would allow a stronger robustness to eavesdroppers attacks and consequently longer distance in a secure communication.

In the last years, the deterministic generation of single photons on demand has become a field of huge interest for quantum cryptography applications. In particular, the fluorescence of a single quantum emitter is the most efficient way to obtain a non-classical light beam with single photons triggered by the excitation pulses. Indeed on-demand single photon generation has been demonstrated from single atoms in microcavities [1], single molecules [2], single quantum dots [3], single quantum dots in strong [4] and week coupled systems [5] and nitrogen vacancies in diamond [6]. Among these proposals, single photon sources based on colloidal semiconductor nanocrystals (NCs) $[7,8]$ have attracted a growing interest as a very promising quantum sources for quantum cryptography [9]. This is due to their unique properties such as high quantum efficiency and, most remarkably, room temperature operation, not possible with alternative technologies such as Stranski-Krastanov quantum dots.

After an introduction on charge dynamic in semiconductor nanocrystals and on the physics of single photon emission, this work reviews our recent results on the fabrication of single photon sources based on novel colloidal NCs and their coupling with photonic nanocavities.

*massimo.devittorio@unisalento.it ; ferruccio.pisanello@unisalento.it .

Quantum Dots and Nanostructures: Synthesis, Characterization, and Modeling VIII,

edited by Kurt G. Eyink, Frank Szmulowicz, Diana L. Huffaker, Proc. of SPIE Vol. 7947,

$794709 \cdot$ ? 2011 SPIE · CCC code: 0277-786X/11/\$18 - doi: 10.1117/12.880575 


\section{COLLOIDAL NANOCRYSTALS AS EFFICIENT SINGLE PHOTON EMITTERS}

Colloidal NCs are a particular type of semiconductor quantum dots (QDs). These nanometer-sized light emitters are synthesized trough wet-chemistry approaches and allow carriers (electrons and holes) to be strongly confined in three dimensions in both valence and conduction bands. NCs are good candidates for photonic applications in different fields such as health, energy, environment and aerospace due to their low fabrication cost, high quantum efficiency at room temperature, high versatility in the chemical synthesis, and broad tunable emission range [10]. A typical structure of a semiconductor NC is sketched in Figure 1A: in order to increase the quantum efficiency of the system, a nanometersized core (which confines the carriers) is usually surrounded by a crystalline shell with a wider bandgap, with the purpose to isolate the structure from the environment. When compared to other types of QDs, colloidal NCs present smaller dimensions and a more regular shape. For instance, Stransky-Krastanov QDs [11], obtained using facilities such as Metal-Organic Chemical Vapor Deposition (MOCVD) or Molecular Beam Epitaxy (MBE), have usually pyramidal shapes, with heights of few nanometers and lateral sizes between $10 \mathrm{~nm}$ and $50 \mathrm{~nm}$. On the contrary colloidal NCs present a spherical shape with a diameter tunable between $2 \mathrm{~nm}$ and $6 \mathrm{~nm}$, thus improving carriers confinement and their mutual interactions. This enhanced carriers localization leads to new physical phenomena that characterize NCs photoluminescence, such as fluorescence blinking and spectral diffusion [12-14]. Indeed, these phenomena have been related to the so-called "non-radiative Auger recombination" [15], that, moreover, allows NCs to emit single photons also when more then one exciton is present in the nanocrystal.

A
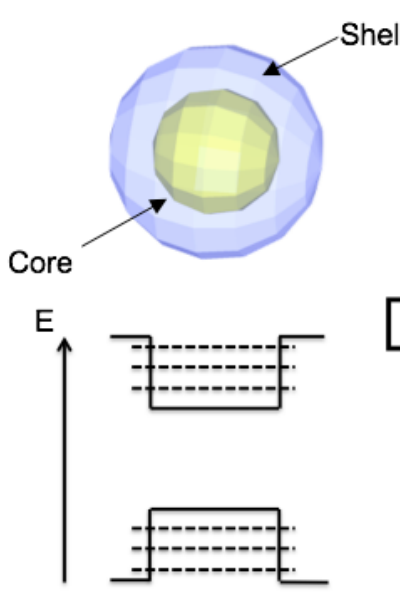
Shell
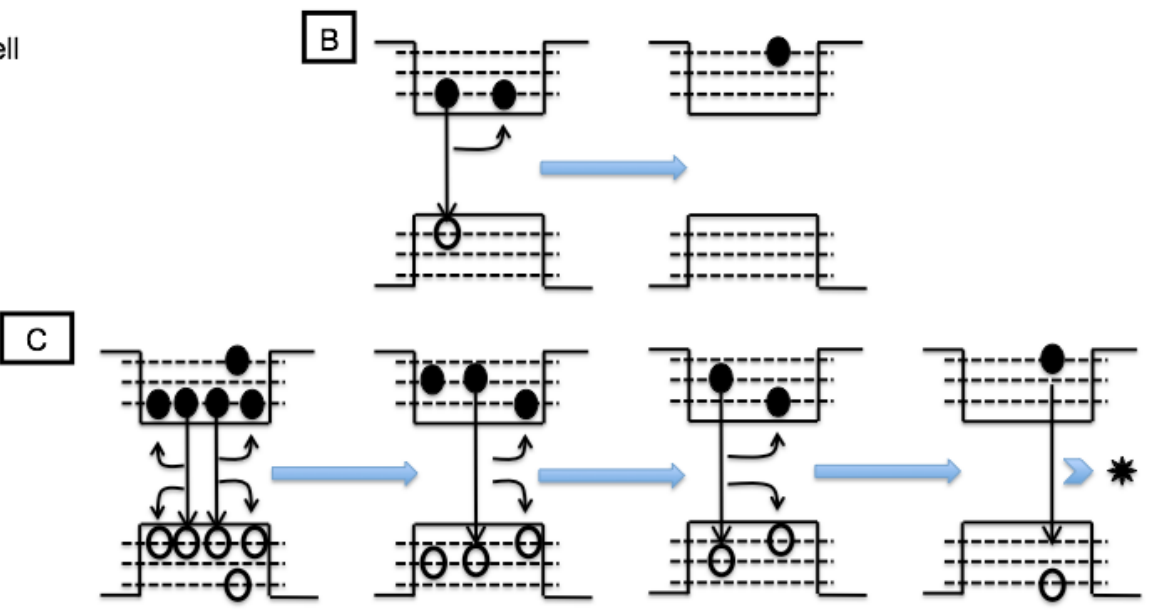

Figure 1 (A) Schematic representation of a colloidal nanocrystal and its bandstructure. (B) Non-radiative Auger recombination in the case of a charged nanocrystal. (C) Non-radiative Auger recombination when more then one e-h pair is excited in the nanocrystal

\subsection{The Auger non-radiative recombination process in colloidal nanocrystals}

As already mentioned, carrier-carrier interactions in colloidal NCs are enhanced by virtue of the high confinement induced by the small dimensions, allowing non-radiative Auger recombination process efficiency to rise up, becoming efficient also at room temperature. Being the cause of blinking, spectral diffusion and single photon emission, Auger effect represents one of the most interesting phenomena in these nanoparticles. It relies in the energy transfer from an electron-hole (e-h) pair recombination to other carriers in someway present in the $\mathrm{NC}$ [16,17]. Two important situations in which it takes place are schematized in Figures 1B and 1C. If a $\mathrm{NC}$ is initially ionized (for example because of thermal- or photo-ionization) and an e-h pair is excited in the nanoparticle, the Auger process constrains the e-h pair to recombine in a non-radiative way by transferring its energy to the localized charge (Figure 1B). Instead, if more than one e-h pair are excited in the NC (Figure 1C), the carrier pairs recombine trough an Auger process and transfer their energy one to each other up to when just one remains excited in the NC. Subsequently, the two charges rapidly relax to their ground states in a couple of picoseconds and are allowed to recombine emitting a photon. From these considerations the picture that can be drawn out is the following. If the Auger recombination is efficient, a charged $\mathrm{NC}$ cannot emit, thus 
leading to the PL fluctuations know as blinking. If instead the NC is not initially charged and more then one e-h pair are excited in the structure, they have a very high probability to recombine non-radiatively through this Auger mechanism until just a single e-h pair remains in the $\mathrm{NC}$, that can recombine through the emission of a photon.

It is thus evident that Auger recombination takes an important role in charge dynamic in colloidal nanocrystals and it is, at the same time, the cause of drawbacks (for instance blinking and spectral diffusion) and physical phenomena at the base of the room-temperature operation of these nanoparticles as single photon emitters. Recently, several ways have been proposed to tailor the efficiency of Auger effect, to engineer NCs spontaneous emission and to reduce blinking [1820]. These strategies can be substantially divided in two types; one relies in tailoring nanocrystals shape and size in order to engineer electrons and holes wavefunctions, while the other one foresees the coupling of NCs with photonic nanostructures $[18,21,22]$. In the following we present two approaches based on these strategies and that could be considered a fist step toward the exploitation of NCs as SPSs for quantum information processing.

\section{OPTICAL CARACTERIZATION OF SINGLE PHOTON SOURCES}

The SPSs described in this paper have been analyzed by time and polarization resolved single molecule spectroscopy. The excitation laser (circularly polarized, picosecond pulsed at a wavelength of $404 \mathrm{~nm}$ ) was focused on a single emitter by a high numerical aperture $(\mathrm{NA}=0.95)$ air-objective. The emission was collected by the same objective and sent into a standard high-sensitivity Hanbury-Brown and Twiss (HBT) setup based on two avalanche photodiodes, which allows to measure directly the autocorrelation function of the fluoscence $I(t)$, i.e

$$
g^{(2)}(\tau)=\frac{<I(t+\tau) I(t)>}{<I(t+\tau)><I(t)>}
$$

Figure 2A shows the scheme of the Hanbury Brown and Twiss setup. Single photons impinging on a beam splitter are split towards the two APDs, which allow to measure $g^{(2)}(t)$ by detecting the delay between the arrival events. The absence of coincident detection events on the two detectors is the fingerprint of a one-by-one photon emission, i.e., photon antibunching. When using a pulsed excitation, the second-order correlation function consists of an array of short peaks, with the period of the pumping sources, and the evidence of single photon emission is the absence of the peak at zero time delay (Figure 2B).
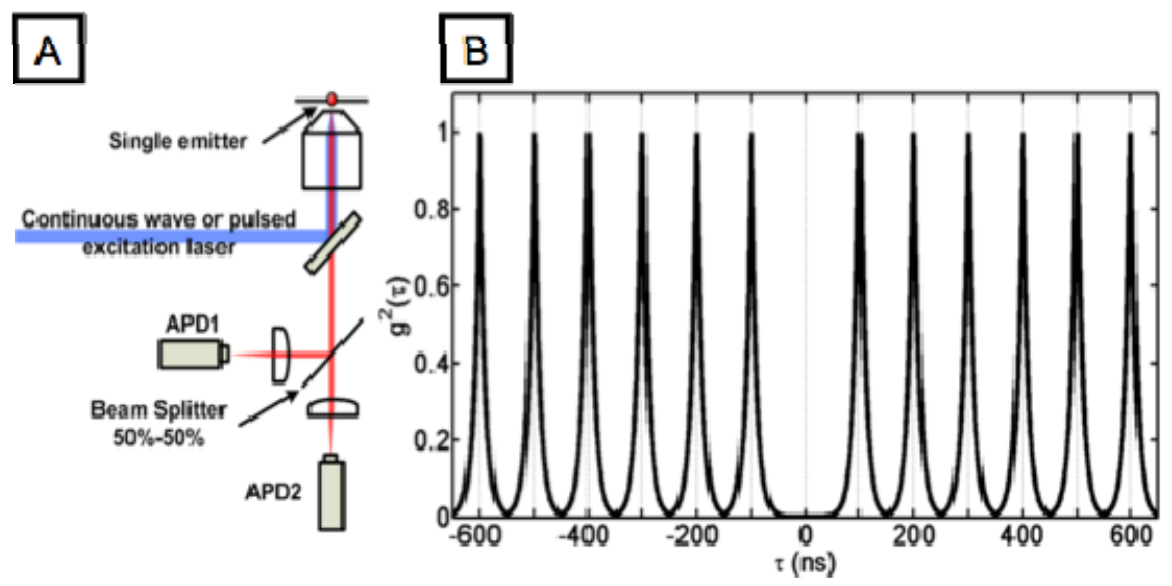

Figure 2 (A) Schematic representation of the Hanbury Brown and Twiss setup. (B) Typical behaviour of the second order autocorrelation function for pulsed excitation.

\section{POLARIZED SINGLE PHOTON EMISSION FROM A COLLOIDAL DOT-IN-ROD}

A strong advantage of colloidal nanocrystals is that by modifying their geometry it is possible to tune their emission properties. It is well known that this can be done in terms of emission wavelength by playing on core and shell size [10], but there are more ways to improve or tune their optical properties. Indeed in past years the full control on the growth parameters in the wet chemical synthesis process was exploited to synthesize different NCs such as rods, tetrapods and 
dimers [23-27] also with interesting polarization properties of the emitted light. Moreover, it has been demonstrated that by keeping a spherical core and changing the size and the shape of shell is also possible to induce a delocalization of just one carrier wavefunction in order to tailor the efficiency of the Auger process or induce a polarization along one of the crystalline axes of the structure. Most of these nanostructures demonstrated to be effective sources of single photons and, in particular CdSe/CdS dot-in-rod (DR) obtained by surrounding a spherical CdSe core with an elongated CdS shell [28, 29] (see sketch in Figure 3A and TEM image in Figure 3B), appear to be very promising quantum emitters, by virtue of their relatively short lifetime and electrical dipole-moment oriented along the rod axis which leads to a high degree of linear polarization [9,30,31]. Indeed, autocorrelation function measurements, reported in Figures 3D, show that when a single DR is excited by picosecond pulses, the probability to emit more then one photon per pulse is $\sim 2 \%$ and that the lifetime of the excited state is $\sim 12 \mathrm{~ns}$. Moreover, polarization resolved measurements performed on a single nanoparticle (Figure 3C) show a degree of linear polarization of about $85 \%$. This feature, invaluable for a SPS device, allows a deterministic photon polarization to be achieved, as required in BB84 and B92 cryptographic algorithms [32-35], without recurring to external polarizers to encode information. In Ref. 9, it is shown that the polarization control of single photons can be achieved by using DRs rotated at different angles, envisioning a strategy to develop polarization controlled, low cost and highly efficient room temperature single photon sources for quantum cryptography applications.

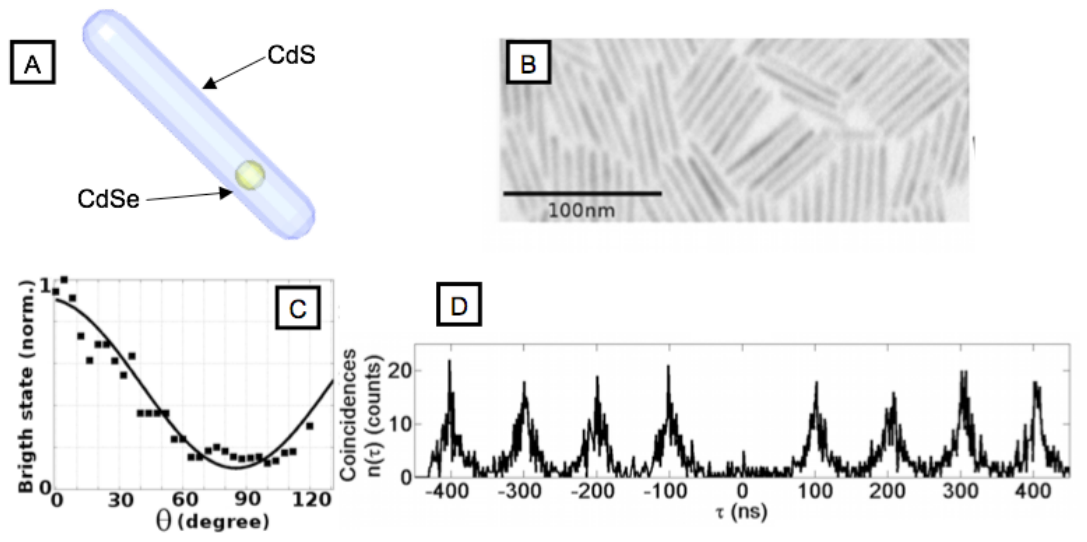

Figure 3 (A) Sketch of a colloidal DR. (B) Transmission Electron Microscope (TEM) image of colloidal dot-in-rods. (C) Polarization resolved photoluminescence of a colloidal DR. (D) Autocorrelation function collected for a single DR

\section{SINGLE PHOTON GENERATION FROM A NANOCRYSTAL PINPOINTED IN A NANOCAVITY}

The presence of a cavity strongly affects the density of available photon states (PDOS) of the environment. When an emitter is placed inside a cavity, many properties of its light emission can be strongly modified: spontaneous emission rate and radiative decay can be engineered and enhanced, radiative pattern and divergence angles can be modified, spectral emission can be narrowed and the emission polarization can be controlled.

When an emitter is weakly coupled to an optical cavity, the spontaneous emission rate of the whole system is given by the Purcell factor [36]

$$
F_{p}=\frac{3}{4 \pi^{2}} \frac{Q}{V}\left(\frac{\lambda}{n}\right)^{3} .
$$

where $\lambda$ is the resonant wavelength and $\mathrm{Q}$ and $\mathrm{V}$ the quality factor and the modal volume of the localized mode, respectively. If $F_{p}>1$, the emitter radiates faster in the cavity than in free space. However the coupling is maximized just when the emitter is placed in the maximum of the electric field intensity. This condition is hard to be fulfilled for pointlike emitters, since it requires a high accuracy in nanocrystals positioning inside an ultra-small volume.

Recently, a new approach has made possible the pinpointing of single colloidal quantum dots by direct electron beam lithography. The controlled localization of ordered arrays of single colloidal nanocrystals was demonstrated in ref.37 by dispersing a specific concentration of nanocrystals inside a negative high-resolution electron-beam resist after 
precipitation and re-dilution in methyl-isobutyl-ketone (MIBK) solvent. Ensembles of nanocrystals embedded in electronic resists can be easily patterned by means of traditional lithographic processes, since the presence of semiconductor clusters in the matrix does not significantly affect the sensitivity of the polymeric host and, at the same time, the emission properties of the nanocrystals are not influenced by the interaction with the electron beam. To obtain on average one nanocrystal inside a nanosized pillar of the $\mathrm{NC} /$ resist blend, direct e-beam patterning is employed (Figures 4A and 4B). Through a careful control of molar density of the NCs dispersed in the resist and pillar volume, i.e., blend layer thickness and pillar diameter, it is possible to obtain a very high probability of having localized single colloidal NCs, whose photon antibunching behavior was confirmed through a confocal microscope and HBT measuremets.

An approach to localize a single $\mathrm{NC}$ in a photonic cavity, described in detail in ref.22, relies in the exploitation of the above-described localization technique on top of a $\mathrm{SiO} 2 / \mathrm{TiO} 2 \mathrm{Bragg}$ mirror. Subsequently, the array of localized single quantum emitters can be clad by a second DBR consisting of four alternating quarter-wavelength thick layers of $\mathrm{TiO} 2 / \mathrm{SiO} 2$. Figures $4 \mathrm{C}$ shows the schematic of the fabricated microcavity and the antibunching demonstration (Figure 4D) from single nanocrystals in microcavity at room temperature. As shown ref.22 this method allow to obtain a Purcell factor of $\sim 2.4$, approaching the theoretical predictions [38].

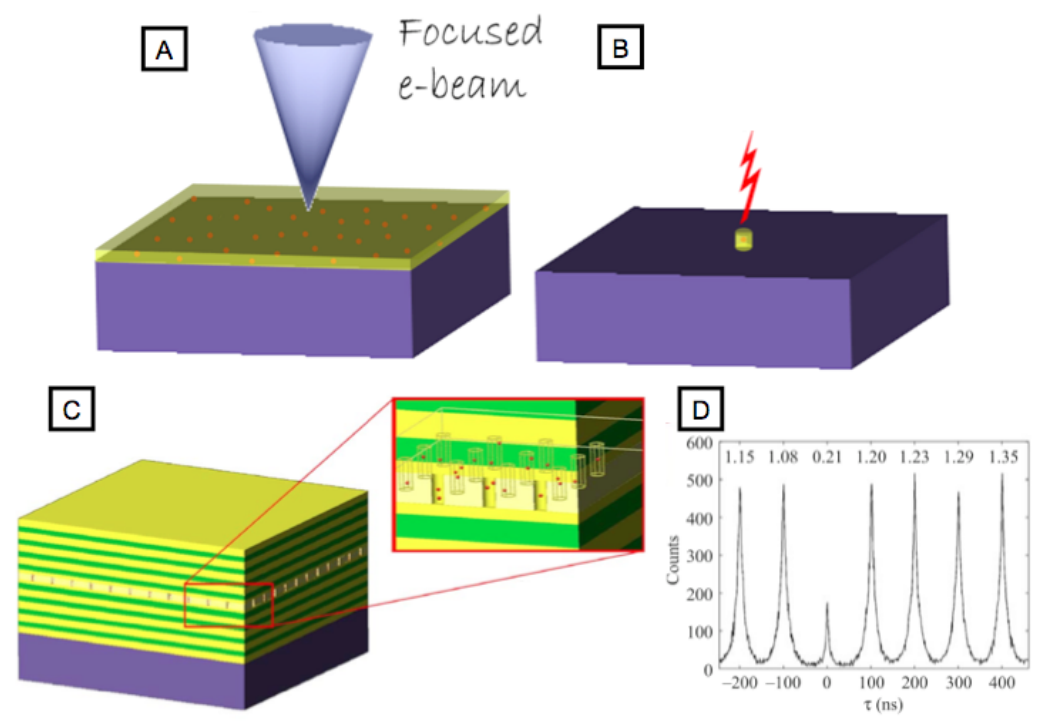

Figure 4 (A) Schematic representation of the localization of single colloidal quantum dots by direct electron beam lithography of a resist/NCs blend. (B) By a careful control of molar density of the NCs dispersed in the resist and pillar volume it is possible to isolate single QDs. (C) Schematic of single colloidal quantum dots microcavity array obtained by direct electron beam lithography and $\mathrm{SiO} 2 / \mathrm{TiO} 2 \mathrm{DBR}$ deposition (D) Histogram of coincidence counts of fluorescence from a single QD in microcavity.

\section{CONCLUSIONS}

In summary the colloidal NC technology allow the realization of advanced single photon devices, which properties can be tailored and engineered by following two parallel approaches. One relies in modifying their geometry in order to model their band structure and induce defined polarization properties, while the other consists in localizing a single nanocrystal in a photonic cavity, thus enhancing the spontaneous emission rate of the emitter. Despite the impressive results reported in literature, many issue still need to be faced and some of the technological problems to be solved, such as the localization of a single nanocrystal in systems more complicated than a DRB-based nanocavity. However, the results reported in this paper coupled to the body of work already reported in literature, make triggered sources of single photons closer and closer to their practical application in quantum communication and quantum cryptography. 


\section{ACKNOWLEDGMENT}

This work was supported by the Italian Ministry of Education, University and Research (MIUR), in the framework of the project "FIRB - Hub di ricerca italo-giapponese sulle nanotecnologie". The authors thank Angela Fiore, Benedetta Antonazzo and Rosanna Mastria for their support in the synthesis of colloidal nanocrystals and Gianmichele Epifani, Gianvito De Iaco and Angelo Melcarne for their expert technical help.

\section{REFERENCES}

[1] Hennrich, M., Legero, T., Kuhn, A., and Rempe, R., "Photon statistics of a non-stationary periodically driven single-photon source,” New J. Phys. 6, 86 (2004)

[2] Brunel, C., Lounis, B., Tamarat, P., and Orrit, M., "Triggered Source of Single Photons based on Controlled Single Molecule Fluorescence," Phys. Rev. Lett. 83, 2722-2725 (1999)

[3] Kako, S., Santori, C., Hoshino, K., Go tzinger, S., Yamamoto, Y. and Arakawa, Y. , "A gallium nitride singlephoton source operating at $200 \mathrm{~K}$ ", Nature Mater. 5, 887-892 (2006).

[4] Englund, D., Faraon, A., Zhang, B., Yamamoto, Y., and Vuckovic, J., "Generation and transfer of single photons on a photonic crystal chip," Opt. Express 15, 5550-5558 (2007)

[5] Faraon, A., Fushman, I., Englund, D., Stoltz N., Petroff, P., and Vukovic, J. "Coherent generation of nonclassical light on a chip via photon-induced tunnelling and blockade," Nature Physics 4, 859-863 (2008).

[6] Simpson, D. A., Ampem-Lassen, E., Gibson, B. C., Trpkovski, S., M. Hossain, F., Huntington, S. T., Greentree, A. D., Hollenberg, L. C. L., and Prawer, S., "A highly efficient two level diamond based single photon source," Appl. Phys. Lett. 94, 203107 (2009).

[7] Michler, P., Imamoglu, A., Mason, M. D., Carson, P. J., Strouse, G. F., and Buratto, S. K., "Quantum correlation among photons from a single quantum dot at room temperature," Nature 406, 968-970 (2000).

[8] De Vittorio, M., Pisanello, F., Martiradonna, L., Qualtieri, A., Stomeo, T., Bramati, A., and Cingolani, R., "Recent advances on single photon sources based on single colloidal nanocrystals", Opto-Electron. Rev. 18, 1-9 (2010).

[9] Pisanello, F., Martiradonna, L., Lemenager, G., Spinicelli, P., Fiore, A., Manna, L., Hermier, J.-P., Cingolani, R., Giacobino, E., De Vittorio, M., and Bramati, A., "Room temperature-dipolelike single photon source with a colloidal dot-in-rod" Appl. Phys. Lett. 96, 033101 (2010).

[10] Murray, C. B., Norris, D.J., and Bawendi, M.G., "Synthesis and characterization of nearly monodisperse CdE (E = sulfur, selenium, tellurium) semiconductor nanocrystallites", J. Am. Chem. Soc. 115, 8706-8715 (1993)

[11] Stranski, I. N., and Von Krastanow, L., "Abhandlungender Mathematisch Naturwissenschaftlichen Klasse", Akademie der Wissenschaften und der Literatur in Mainz 146, 797 (1939).

[12] Nirmal, M., Dabbousi, B. O., Bawendi, M.G., Macklin, J.J., Trautman, J.K., Harris, T.D., Brus, L.E., "Fluorescence intermittency in single cadmium selenide nanocrystals", Nature 383, 802-804 (1996).

[13] Empedocles, S.A., Norris, D.J., and Bawendi, M.G., "Photoluminescence Spectroscopy of Single CdSe Nanocrystallite Quantum Dots," Phys. Rev. Lett. 77, 3873-3876 (1996).

[14]Lu H.P., and Xie, X.S., "Single-molecule spectral fluctuations at room temperature," Nature 385, 143-146 (1997).

[15] Brokmann, X., Messin, G., Desbiolles, P., Giacobino, E., Dahan M., and Hermier, J. P., "Colloidal CdSe/ZnS quantum dots as single-photon sources," New J. Phys. 6, 99 (2004).

[16] Klimov, V. I., Mikhailovsky, A., McBranch, D., Leatherdale C. A., and Bawendi M. G., "Quantization of multiparticle Auger rates in semiconductor quantum dots," Science 287, 1011-1013 (2000).

[17] Spinicelli, P., Buil, S., Quélin, X., Mahler, B., Dubertret, B., and Hermier, J.-P., "Bright and Grey States in CdSe-CdS Nanocrystals Exhibiting Strongly Reduced Blinking”, Phys. Rev. Lett. 102, 136801 (2009).

[18] Pisanello, F., Qualtieri, A., Stomeo, T., Martiradonna, L., Cingolani, R., Bramati, A., and De Vittorio, M., "High-Purcell-factor dipolelike modes at visible wavelengths in H1 photonic crystal cavity," Opt. Lett. 35, 1509-1511 (2010).

[19] Mahler, B., Spinicelli, P., Buil, S., Quelin, X., Hermier, J.-P., and Dubertret, B., "Towards non-blinking colloidal quantum dots,"Nature Mater. 7, 659-664 (2008).

[20] Wang, X., Ren, X., Kahen, K., Hahn, M. A., Rajeswaran, M., Maccagnano-Zacher, S., Silcox, J., Cragg, G. E., Efros, A. L., and Krauss, T. D., "Non-blinking semiconductor nanocrystals," Nature 459, 686 (2009). 
[21] Vion, C., Spinicelli, P., Coolen, L., Schwob, C., Frigerio, J.-M., Hermier, J.-P., and Maître, A., "Controlled modification of single colloidal CdSe/ZnS nanocrystal fluorescence through interactions with a gold surface," Opt. Express 18, 7440 (2010) .

[22] Qualtieri, A., Morello, G., Spinicelli, P., Todaro, M. T., Stomeo, T., Martiradonna, L., De Giorgi, M., Quélin, X, Buil, S., A. Bramati, Hermier, J.-P., Cingolani, R., and De Vittorio, M., "Nonclassical emission from single colloidal nanocrystals in a microcavity: a route towards room temperature single photon sources", New J. Phys. 11, 033025 (2009).

[23] Hu, J., Li, L.S., Yang, W., Manna, L., Wang, L.W., and Alivisatos, A.P., "Linearly polarized emission from colloidal semiconductor quantum rods," Science 292, 2060-2063 (2001).

[24] Talapin, D.V., Koeppe, R., Go tzinger, S., Kornowski, A., Lupton, J.M., Rogach, A.L., Benson, O., Feldmann, J., and Weller, H., "Highly emissive colloidal CdSe/CdS heterostructures of mixed dimensionality", Nano. Lett. 3, 1677 (2003).

[25] Fiore, A. Mastria, R., Lupo, M.G., Lanzani, G., Giannini, C., Carlino, E., Morello, G., De Giorgi, M., Li, Y. Cingolani, R., and Manna, L., "Tetrapod-shaped colloidal nanocrystals of II-VI semiconductors prepared by seeded growth,” J. Am. Chem. Soc. 131, 2274-2282 (2009).

[26] Manna, L., Scher, E., and Alivisatos, A.P., "Synthesis of soluble and processable rod, arrow, teardrop, and tetrapod shaped CdSe nanocrystals," J. Am. Chem. Soc. 122, 12700-12706 (2000).

[27] Liddell C.M., and Summers, C.J., "Monodispersed ZnS dimers, trimers, and tetramers for lower symmetry photonic crystal lattices", Adv. Mater. 15, 1715-1719 (2003).

[28] Carbone, L., Nobile, C, De Giorgi, M., Della Sala, F., Morello, G., Pompa, P., Hytch, M., Snoeck, E., Fiore, A., Franchini, I. R., Nadasan, M., Silvestre, A. F., Chiodo, L., Kudera, S., Cingolani, R., Krahne, R., and Manna, L. "Synthesis and Micrometer-Scale Assembly of Colloidal CdSe/CdS Nanorods Prepared by a Seeded Growth Approach," Nano Lett. 7, 2942 (2008).

[29] Talapin, D. V., Koeppe, R., Go tzinger, S., Kornowski, A., Lupton, J. M., Rogach, A. L., Benson, O., Feldmann, J., and Weller, H. "Highly Emissive Colloidal CdSe/CdS Heterostructures of Mixed Dimensionality," Nano Lett. 3, 1677-1681 (2003).

[30] Pisanello, F., Martiradonna, L., Spinicelli, P., Fiore, A., Hermier, J.-P., Manna, L., Cingolani, R., Giacobino, E., De Vittorio, M., and Bramati, A., "Dots in rods as polarized single photon sources," Superlattices Microst. 47, 165-169, (2009).

[31] Pisanello, F., Leménager, G., Martiradonna, L., Spinicelli, P., Fiore, A., Amo, A., Giacobino, E., Cingolani, R., De Vittorio, M., and Bramati, A., "Evaluation of oscillator strength in colloidal CdSe/CdS dots-in-rods," Phys Status Solidi C 7, 2688-2691 (2010).

[32] Bennett C. H., and Brassard, G., Int. Conf. on Computers, Systems and Signal Processing, Bangalore, India, 175 (1984).

[33] Bennett, C.H., Bessette, F., Brassard, G., Salvail, L., and Smolin, J., “Experimental quantum cryptography," J. Cryptol. 5, 3-28 (1992).

[34] Bennet, C.H., "Quantum cryptography using any two nonorthogonal states," Phys. Rev. Lett. 68, 3121-3124 (1992).

[35] Pisanello, F., Martiradonna, L., Spinicelli P., Fiore, A., Hermier, J.P., Manna, L., Cingolani, R., Giacobino, E., Bramati, A., and De Vittorio, M., "Polarized single photon emission for quantum cryptography based on colloidal nanocrystals", IEEE Proc. of 11th Int. Conf. on Transparent Optical Networks, 1-4 (2009).

[36] Purcell, E.M., "Spontaneous emission probabilities at radio frequencies," Phys. Rev. 69, 681 (1946).

[37] Qualtieri, A., Morello, G., Spinicelli, P., Todaro, M. T., Stomeo, T., Martiradonna, L., De Giorgi, M., Quelin, X., Buil, S., Bramati, A., Hermier, J. P., Cingolani, R., and De Vittorio, M., "Room temperature single-photon sources based on single colloidal nanocrystals in microcavities," Superlattices Microst. 47, 187-191 (2010).

[38] Yokoyama, H., "Physics and Device Applications of Optical Microcavities," Science 256, 5053 (1992). 